\title{
RENDA E CONDIÇÕES DE VIDA NOS MUNICÍPIOS DE MINAS GERAIS: POSSIBILIDADES A PARTIR DA REGRESSÃO GEOGRAFICAMENTE PONDERADA (GWR)
}

Júlio César Tavares de Paiva Silva

\section{Resumo:}

Nas últimas décadas, o desenvolvimento e utilização de novas ferramentas e métodos estatísticos e computacionais permitiram que pesquisadores pudessem compreender diversos tipos de fenômenos, entre eles os socioeconômicos e espaciais. Nesse sentido, a partir de técnicas de estatística espacial e geoprocessamento, o presente trabalho objetiva analisar a relação entre renda e alguns indicadores sociais (mortalidade infantil, analfabetismo e porcentagem de pobres) nos municípios de Minas Gerais com base nos dados do último Censo Demográfico (2010). Considerando os resultados do modelo de regressão espacial desenvolvido, percebe-se que, de fato existe uma forte correlação entre renda e melhores condições de vida. De modo geral, quanto maior a renda média nos municípios, menores (ou melhores) são os seus indicadores sociais de vulnerabilidade.

Palavras chave: Estatística espacial; indicadores socioeconômicos; Minas Gerais. 


\section{1- INTRODUÇÃO}

Nas últimas décadas, a geografia quantitativa avançou significativamente permitindo aos geógrafos o desenvolvimento e utilização de novas ferramentas e métodos em suas pesquisas. Ao mesmo tempo, melhorias na tecnologia da informação e da computação permitiram que profissionais de diversas áreas compreendessem e solucionassem os mais variados tipos de problemas. Nesse sentido, o desenvolvimento e aperfeiçoamento dos Sistemas de Informação Geográfica (SIG) e das técnicas de processamento de dados georreferenciados (Geoprocessamento) a partir da década de 1970 foi fundamental. Fenômenos climáticos, geológicos, geomorfológicos, humanos, socioeconômicos, entre outros, puderam ser melhor compreendidos em busca de identificação de padrões de ocorrência, respostas e soluções (CÂMARA et al, 1996; BARBETTTA, 2012; ROGERSON, 2012).

Em especial, a estatística aplicada aos estudos socioeconômicos permite que pesquisadores conheçam as características das populações, seus desejos e necessidades, o que facilita na tomada decisões em relação à elaboração de políticas públicas pelo poder público. De acordo com Ignácio (2010), os estudos geoestatísticos são de suma importância e todos os governos possuem departamentos oficiais destinados à produção e análises deste tipo de informação. No Brasil, o principal responsável por tais pesquisas é o Instituto Brasileiro de Geografia e Estatística - IBGE criado em 1939 (IBGE, 2010). Dados e informações como mortalidade infantil, morbidade, nível educacional, pobreza, renda domiciliar, renda per capita, acesso à saneamento básico, entre diversos outros são cruciais para se conhecer o perfil da população nacional em diferentes escalas (estados, municípios, regiões, áreas urbanas, etc.).

Nesse sentido, a combinação de indicadores sociais e econômicos permitem ao pesquisador analisar a realidade e a qualidade de vida de uma população. Minas Gerais apresenta o maior número de municípios no Brasil totalizando 853 distribuídos em 12 mesorregiões que abrigavam em 2010 mais de 19 milhões de habitantes (IBGE, 2010). De acordo com Júnior (2007), a heterogeneidade entre as regiões e municípios mineiros é marcante por conta da grande extensão territorial do Estado, que contribui para significativas diferenças climáticas, geológicas e geomorfológicas, culturais, industriais e econômicas. Assim, a análise de alguns indicadores socioeconômicos se faz necessária para entender como os municípios mineiros se comportam em relação à vulnerabilidade social e os rendimentos econômicos.

Nesse contexto, o presente trabalho objetiva analisar a relação entre renda e indicadores sociais (mortalidade infantil, analfabetismo e porcentagem de pobres) nos municípios mineiros 
com base nos dados do último Censo Demográfico (2010). A hipótese investigada, a partir da aplicação de métodos de estatística espacial e de técnicas de geoprocessamento, é que a renda não se materializa necessariamente em melhores condições de vida. Os dados das variáveis utilizadas foram extraídos Atlas do Desenvolvimento Humano no Brasil - 2013, e as bases vetoriais dos municípios e mesorregiões fornecidas pelo IBGE.

\section{2- MÉTODOS E APLICAÇÕES DA ESTATÍSTICA ESPACIAL: UMA BREVE REVISÃO DA LITERATURA}

Segundo Assunção (2007), no campo geográfico toda observação possui referência espacial e temporal. Sendo assim, vários trabalhos foram realizados ao longo do último século para que os estudos estatísticos avançassem para um campo conhecido como estatística espacial. Neste resgate temporal, destacam-se os trabalhos realizados por Student (1914 apud KREMPI, 2004), sobre os métodos quantitativos tradicionais, além dos estudos de Moran 1948 (apud KREMPI, 2004), e Geary (1954 apud KREMPI, 2004), que apresentavam índices para a detecção de autocorrelação espacial em um conjunto de dados.

De modo geral, os processos de análise espacial incluem métodos de seleção, manipulação, investigação de padrão de dados (se apresentam agregação definida ou distribuição aleatória), análise exploratória, confirmatória, entre outros. Um método que se destaca no campo da estatística espacial é a Regressão Espacial, que deriva da Análise de Regressão. Por sua vez, esta pode ser entendida como um estudo que utiliza a relação entre duas ou mais variáveis de modo que uma variável possa ser explicada pela outra ou outras. Já aquela, inclui a variável espaço nesta análise, ou seja, como a variável $\mathrm{X}$ pode ser explicada pela variável $\mathrm{Y}$ em um recorte espacial determinado (KREMPI, 2004; ROGERSON, 2012). Dessa forma, a Regressão Espacial pode ser bastante útil na criação de modelos que representam as relações entre variáveis em determinadas áreas.

Nesse contexto, Araújo et. al. (2010) investigaram a distribuição espacial da mortalidade por homicídio e as desigualdades sociais com base na raça/cor do indivíduo em áreas urbanas em Salvador, Bahia. Através de métodos quantitativos como Índice de Moran Global e Local, além da regressão geograficamente ponderada (GWR), os autores perceberam aumentos na taxa de mortalidade por homicídio em áreas na cidade que predominavam a população negra de 15 a 49 anos. Os resultados foram apresentados através de mapas que identificaram a distribuição da população com base nas características pré-selecionadas. 
Já Gonçalves et. al. (2011), analisaram a distribuição espacial da mortalidade neonatal e os fatores relacionados entre 2000 e 2006 também em Salvador. Através de uma combinação de técnicas de estatística espacial (Índice de Moran) com técnicas de geoprocessamento trabalhadas em SIG, os autores identificaram uma correlação espacial para a mortalidade neonatal. Neste caso, os maiores riscos de mortalidade neonatal estavam concentrados em áreas do centro e subúrbio onde reside a população com baixos índices socioeconômicas.

Em um trabalho mais recente, Magalhães e Medronho (2017), pesquisaram a distribuição espacial da tuberculose e os fatores socioeconômicos condicionantes entre 2005 e 2008 no Rio de Janeiro, também utilizando modelos de regressão espacial. Nesse caso, os autores identificaram na cidade os setores censitários que possuíam maiores ocorrências da doença e relacionaram com variáveis socioeconômicas. Como resultados, os autores perceberam que a população que é mais acometida pela doença é justamente a que possui renda mais baixa, que mora em locais precários como favelas e que não possui acesso à serviços de saúde. Os resultados foram apresentados através de tabelas e mapas que mostraram detalhadamente as relações existentes entre a características observadas e as áreas de estudo.

Com objetivos bem distintos, Araújo et. al. (2014), utilizaram um modelo de regressão espacial para estimar a produtividade da soja com base em variáveis agrometeorológicas no oeste do estado do Paraná, através de métodos de estatística espacial local e global combinados com técnicas de geoprocessamento aplicadas em softwares de SIG. De acordo com os autores, os resultados obtidos pelo modelo de regressão espacial foram significativamente melhores do que o modelo de regressão múltipla clássico.

Por fim, após o levantamento bibliográfico a respeito da utilização de métodos de regressão espacial e estatística espacial em geral, fica claro que tais técnicas são úteis para se fazer análises em diferentes áreas do conhecimento e com diferentes objetivos. Em relação aos trabalhos que envolvem variáveis socioeconômicas, os resultados podem servir para uma melhor compreensão da dinâmica populacional no espaço, além de base para a criação de políticas públicas pelo Estado que busquem oferecer uma melhor qualidade de vida à população.

\section{3- MATERIAIS E MÉTODOS}

De modo geral, os procedimentos metodológicos consistiram em coleta, tratamento, análises exploratórias dos dados, elaboração de mapas temáticos e análise geral dos resultados. 
A Figura 1 apresenta o fluxograma com o resumo da metodologia aplicada:

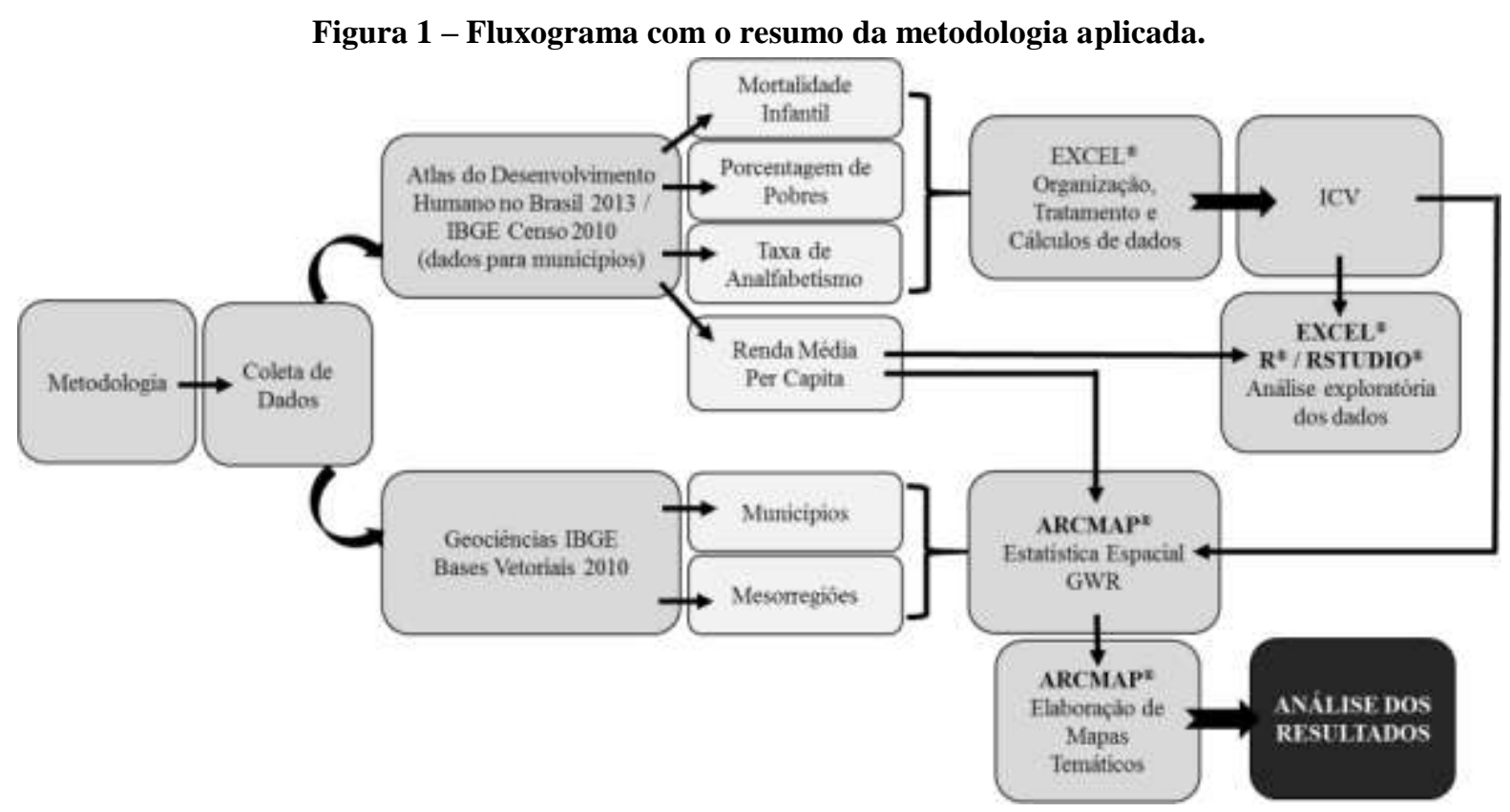

Fonte: Elaborado pelo autor.

Inicialmente, foi necessário criar um indicador que representasse o nível da qualidade de vida da população mineira. Para isso, algumas variáveis foram coletadas a partir do site do Atlas do Desenvolvimento Humano no Brasil. Vale ressaltar que os dados fornecidos pelo Atlas são na verdade do Censo Demográfico realizado pelo IBGE em 2010. As variáveis selecionadas para cada município foram:

- Mortalidade Infantil (MI): crianças que morrem antes de completar 1 ano de idade por mil nascidos vivos.

- Taxa de Analfabetismo (TA): percentual de pessoas com 15 e mais anos de idade que não sabem ler e escrever pelo menos um bilhete simples, no idioma que conhecem.

- $\quad$ Porcentagem de Pobres (PP): percentual de pessoas que viviam em 2010 com renda mensal inferior à 140 reais.

Após a coleta dos dados, estes foram manipulados através do Excel 2013. Primeiramente, os dados foram organizados em colunas (indicadores) e linhas (municípios). Em seguida, alguns cálculos foram realizados a fim de gerar o Indicador de Condições de Vida (ICV). Primeiramente, média geral $(\mu 1)$ e desvio padrão $(\sigma)$ de todos os municípios em cada variável. Logo após, os 
valores das variáveis para cada município foram subtraídos da razão entre a média geral e o desvio padrão de cada variável, gerando três novas variáveis para cada município (Equação 1A, 1B e 1C). Em seguida foi calculada para cada município a média entre as três novas variáveis $(\mu 2)$ geradas no passo anterior (Equação 2).

$$
\begin{aligned}
& \text { Variável } 1=\mathrm{MI}_{\text {município }}-\frac{\mu 1}{\sigma} \quad \text { (Equação 1A) } \\
& \text { Variável } 2=\mathrm{TA}_{\text {município }}-\frac{\mu 1}{\sigma} \quad \text { (Equação 1B) } \\
& \text { Variável } 3=\mathrm{PP}_{\text {municipio }}-\frac{}{\sigma} \quad \text { (Equação 1C) } \\
& \mu 2 \text { município }=\frac{\text { var. } 1+\text { var. } 2+\text { var. } 3}{3} \quad \text { (Equação 2) }
\end{aligned}
$$

Os valores dessas médias ( $\mu 2)$ variavam entre positivos e negativos, o que dificultava a interpretação dos resultados. Sendo assim, foi necessário padronizar os resultados para que se distribuíssem entre 0 e 1 . Nesse caso, os valores mais próximos de zero significam baixo ICV e valores próximos de 1 alto ICV. Para a padronização, foi necessário calcular o máximo e o mínimo dos valores das médias $(\mu 2)$ geradas anteriormente. Por fim, o valor de cada média $(\mu 2)$ dos municípios foi subtraído pelo valor do mínimo e divido pelo valor de cada média subtraído pelo máximo (Equação 3). Os resultados desse último cálculo apresentam o ICV padronizado que varia entre 0 e 1.

$$
\begin{aligned}
& \mu 2 \hat{i} \text { - mínimo (Equação 3) } \\
& \mathrm{ICV}=\frac{}{\mu 2_{\text {municipio }}-\text { máximo }}
\end{aligned}
$$

Após a geração do ICV para cada município mineiro, os dados sobre a Renda média per capita mensal municipal foram coletados também a partir do Atlas do Desenvolvimento Humano no Brasil. No próprio Excel foi gerado o gráfico de dispersão (Gráfico 1) com o resultado da relação entre as variáveis. 


\section{Gráfico 1 - Correlação entre renda média per capita e ICV nos municípios mineiros, 2010}

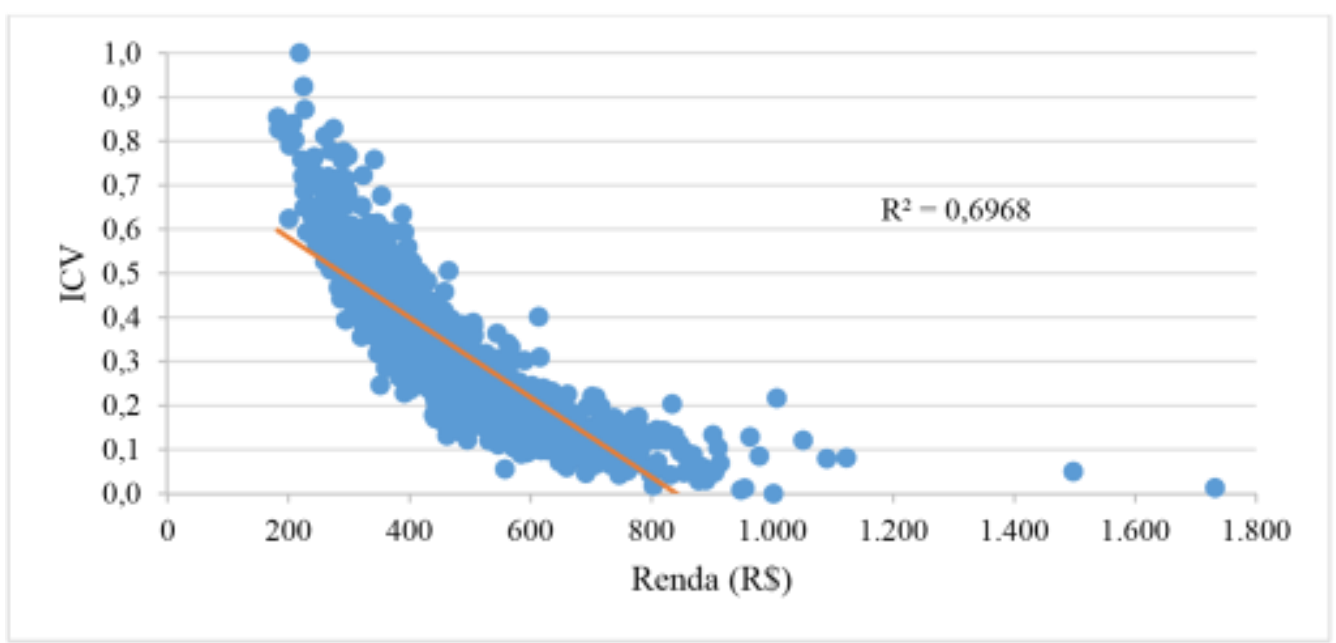

Fonte: Atlas do Desenvolvimento Humano no Brasil - 2013 e IBGE 2010.

Com base no Gráfico 1, nota-se que existe uma significativa correlação entre renda per capita e ICV. O coeficiente de correlação (R2) foi de 0,6968 e a maioria dos municípios localizamse próximos à linha de tendência. Alguns outliers também são observados, tanto no sentido positivo (alta renda e baixo ICV) quanto no sentido negativo (baixa renda e alto ICV). O município de Nova Lima (outlier positivo) na mesorregião metropolitana de Belo Horizonte, apresenta os melhores valores se comparado com todos os outros 852 municípios mineiros. A renda média per capita em 2010 é de $\mathrm{R} \$ 1.731,00$ e o ICV 0,013, ou seja, a grande maioria das pessoas possui renda alta e acesso à recursos de saúde, saneamento e educação. Por outro lado, o município de Santa Helena de Minas (outlier negativo) no Vale do Mucuri, apresenta os piores índices. A renda média é de apenas $\mathrm{R} \$ 218,43$ enquanto que o ICV é 1 . Nesse caso, pode-se inferir que, no geral, as pessoas possuem uma renda baixíssima, baixos níveis de escolaridade e de acesso ao serviços de saúde e saneamento.

$\mathrm{Na}$ segunda parte, os gráficos para a análise exploratória dos dados foram elaborados através dos softwares Excel 2013, R e RStudio (pacote ggplot2). Os dados de ICV e Renda per capita nos municípios foram reorganizados e agrupados com base nas 12 mesorregiões mineiras nas quais cada município pertence. Nestes softwares foram gerados gráficos com o comparativo das variáveis analisadas entre as mesorregiões (Gráficos 2 e 3).

$\mathrm{Na}$ terceira parte, os dados socioeconômicos foram transportados para o software ArcMAP do pacote ArcGIS 10.1. Os dados vetoriais utilizados nessa pesquisa correspondem aos polígonos que representam os limites dos municípios e das mesorregiões mineiras. Ambos foram 
adquiridos junto ao catálogo de downloads de geociências do IBGE. O procedimento inicial consistiu em unir os dados de ICV e Renda trabalhados anteriormente no Excel com a tabela de atributos dos arquivos vetoriais. Para isso, foi utilizada a ferramenta join do ArcGIS que permite a união entre diferentes planilhas a partir de uma variável comum entre elas. Neste caso, a variável comum foi o geocódigo de cada município.

Em seguida, através da ferramenta Spatial Statistics Tools do ArcMap, foi utilizado o modelo Geographically Weighted Regression (GWR) ou Regressão Geograficamente Ponderada a fim de avaliar a predição do ICV (variável dependente), com base na renda (variável explicativa) dos municípios de mineiros. O GWR é uma ferramenta desenvolvida com o objetivo de analisar fenômenos que sofrem variação na área em estudo, advinda da heterogeneidade espacial. De acordo com Rogerson (2012), este método está ligado ao ponto de vista local da regressão. Para cada localização, pode-se estimar uma equação de regressão na qual pesos são atribuídos para as observações vizinhas. Pesos maiores são atribuídos aos vizinhos mais próximos da localização e pesos menores às observações mais distantes.

O algoritmo escolhido para definir a largura de banda ou vizinhança foi o Akaike Information Criterion (AICC) que é uma medida de desempenho útil para comparar diferentes modelos de regressão. Nele é considerado a complexidade do modelo, o que permite um melhor ajuste dos dados observados. Ademais, o AICC também serve para comparar modelos com diferentes variáveis explicativas, aplicadas à mesma variável dependente (LOBO, et. al.).

Em relação aos parâmetros gerados pelo GWR, além dos índices de regressão local $\left(\mathrm{R}^{2}\right)$ calculados para cada município, foram identificados os padrões e a distribuição espacial dos resíduos, que representam os casos de menor e maior poder de explicação do modelo. Por fim, foram elaborados os mapas temáticos representando os resultados também no ArcMap.

Em seguida, os resultados finais foram analisados e discutidos.

\section{4- RESULTADOS E DISCUSSÃO}

A partir dos resultados apresentados no Gráfico 1, percebe-se claramente que existecorrelação entre as variáveis estudadas. De modo geral, quanto maior a renda do município, menor é o seu ICV. Dentro da análise exploratória em escala regional, o comportamento da renda média per capita e do ICV podem ser observados no Gráfico 2. 


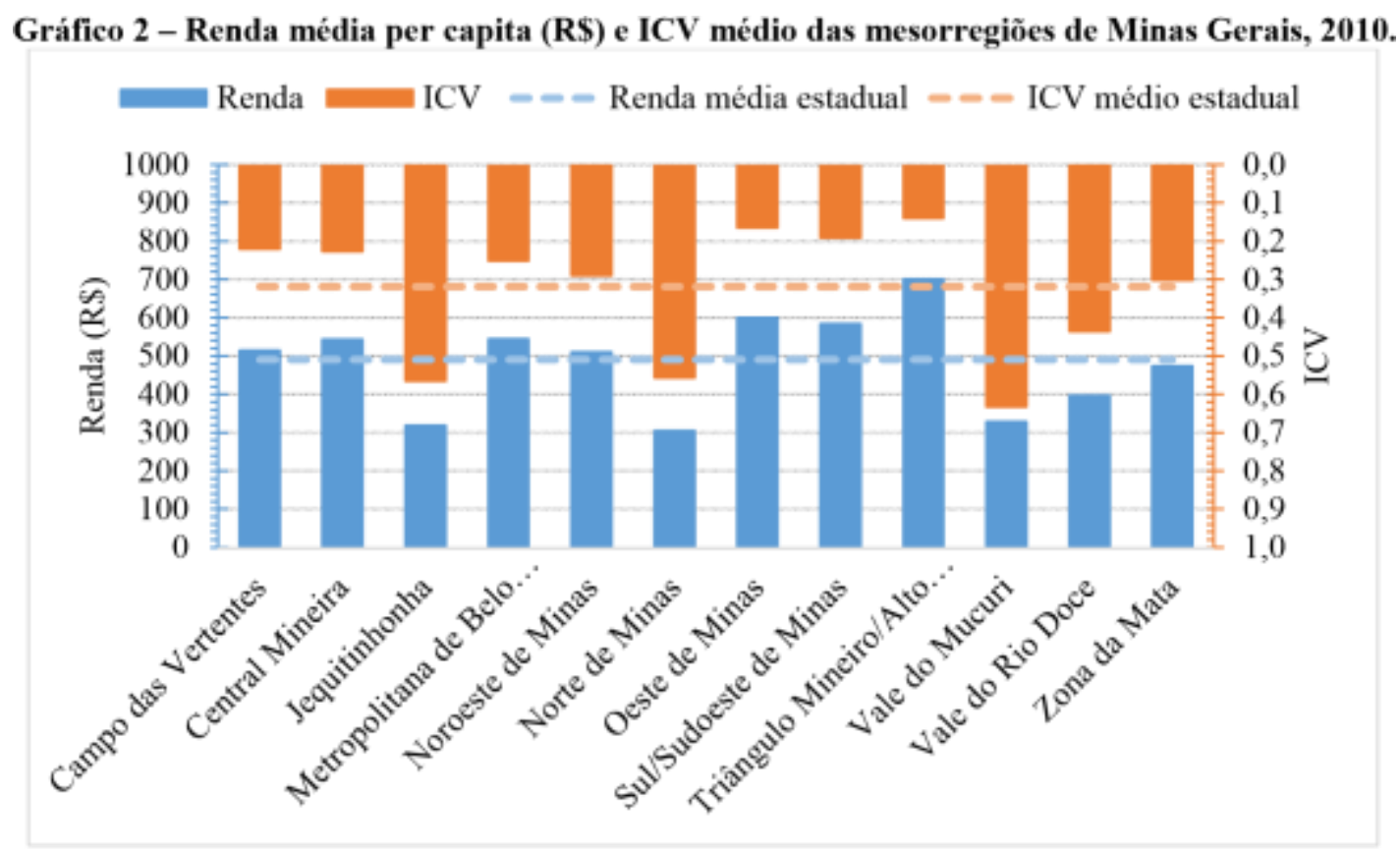

Fonte: Atlas do Desenvolvimento Humano no Brasil - 2013 e IBGE 2010.

No que se refere à renda média per capita, o Triângulo Mineiro/Alto Paranaíba se destaca positivamente com renda média de $\mathrm{R} \$ 701,19$, seguido por Oeste de Minas ( $\mathrm{R} \$$ $600,28)$ e Sul/Sudoeste $(\mathrm{R} \$ 584,13)$. Os destaques negativos ficam por conta do Norte de Minas com média de $\mathrm{R} \$ 304,93$, seguido por Vale do Jequitinhonha ( $\mathrm{R} \$ 319,17)$; Vale do Mucuri ( $\mathrm{R} \$$ 328, 29), e Vale do Rio Doce ( $\mathrm{R} \$ 397,12)$. As demais mesorregiões ficam mais próximas da média do Estado ( $\mathrm{R} \$ 490,59)$. Nesse ponto, é importante ressaltar que o Vale do Rio Doce aparece como uma surpresa negativa visto que a literatura já afirmara que Norte de Minas, Vale do Jequitinhonha e Mucuri historicamente tem baixo desenvolvimento socioeconomico ${ }^{1}$.

Em relação ao ICV, a média estadual é de 0,31 e a correlação com a renda é confirmada para as três mesorregiões mais ricas. O Triângulo Mineiro/Alto Paranaíba apresenta o menor valor $(0,13)$, em seguida Oeste de Minas $(0,16)$ e Sul/Sudoeste $(0,19)$. Do ponto de vista negativo, os valores mais altos também são apresentados pelas mesorregiões mais pobres, entretanto a ordem é alterada. O Vale do Mucuri apresenta um valor de 0,63, seguido por Vale do Jequitinhonha $(0,56)$ e Norte de Minas $(0,55)$. Esta alteração pode ser explicada por conta dos altos valores que dois municípios do Vale do Mucuri apresentam (Santa Helena de Minas 1 e Setubinha 0,92$)$, elevando a média para a mesorregião. O Gráfico 4 pode auxiliar no entendimento da mudança supracitada, além de apresentar a distribuição e o comportamento geral do ICV dos municípios nas mesorregiões.

\footnotetext{
${ }^{1}$ Ver Sawaya et.al (2003); Rosycler (2004); Júnior (2007); Resende (2008).
} 


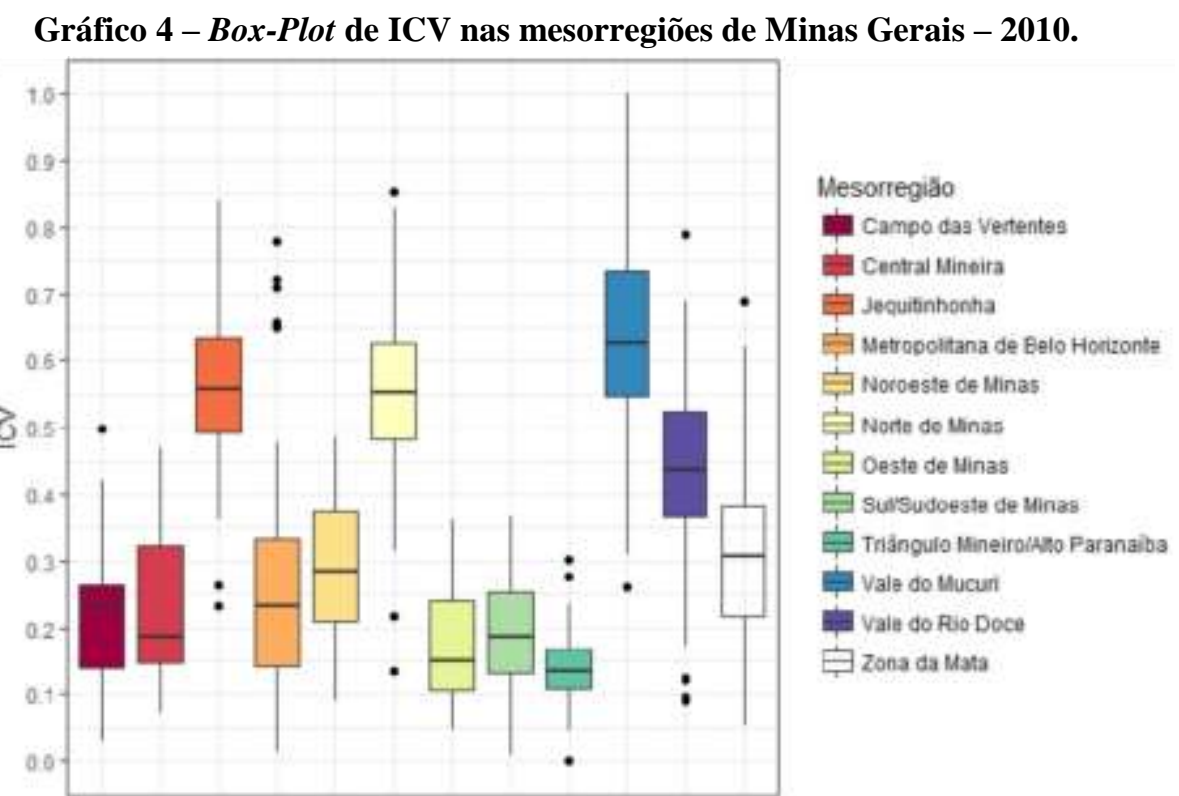

Fonte: Atlas do Desenvolvimento Humano no Brasil - 2013 e IBGE 2010.

No Vale do Mucuri, percebe-se que existe uma grande variação entre os municípios (de 0, 25 em Nanuque a 1 em Santa Helena de Minas). Por outro lado, a distribuição mais homogênea está no Triangulo Mineiro/Alto Paranaíba, o que significa que, no geral, a maior parte dos municípios apresentam boas condições de educação, saúde, saneamento e distribuição de renda. No que diz respeito aos outliers, praticamente todas as mesorregiões apresentam valores discrepantes e a Metropolitana de $\mathrm{BH}$ aparece com o maior número de municípios com valores de ICV bem acima da média regional que é de 0,25 (Congonhas do Norte 0,77, Serra Azul de Minas 0,72, Rio Vermelho 0,70, Alvorada de Minas 0, 65 e Santo Antônio do Itambé 0,64).

Sobre a estatística espacial, as Figuras 2 e 3 representam os resultados. De modo geral, pode-se reafirmar que existe associação direta entre ICV e renda nos municípios mineiros. $\mathrm{O}$ valor de $\mathrm{R}^{2}$ global ajustado, definida a renda como variável explicativa, foi de 0,89 , o que aponta um alto poder explicativo da renda sobre o ICV. Os escores de $\mathrm{R}^{2}$ local também foram altos e corroboram com o apontamento anterior. Dos 853 municípios mineiros, 678 apresentaram valores acima de 0,51. Os municípios mais bem ajustados ao modelo estão concentrados principalmente nas mesorregiões Norte e Vale do Mucuri. Já os que tiveram um baixo ajuste concentram-se no Triangulo Mineiro/Alto Paranaíba e Metropolitana de BH. 
Figura 2 - ICV e Renda média per capita nos municípios e mesorregiões de Minas Gerais, 2010 Regressão Espacialmente Ponderada (GWR): $\mathbf{R}^{2}$

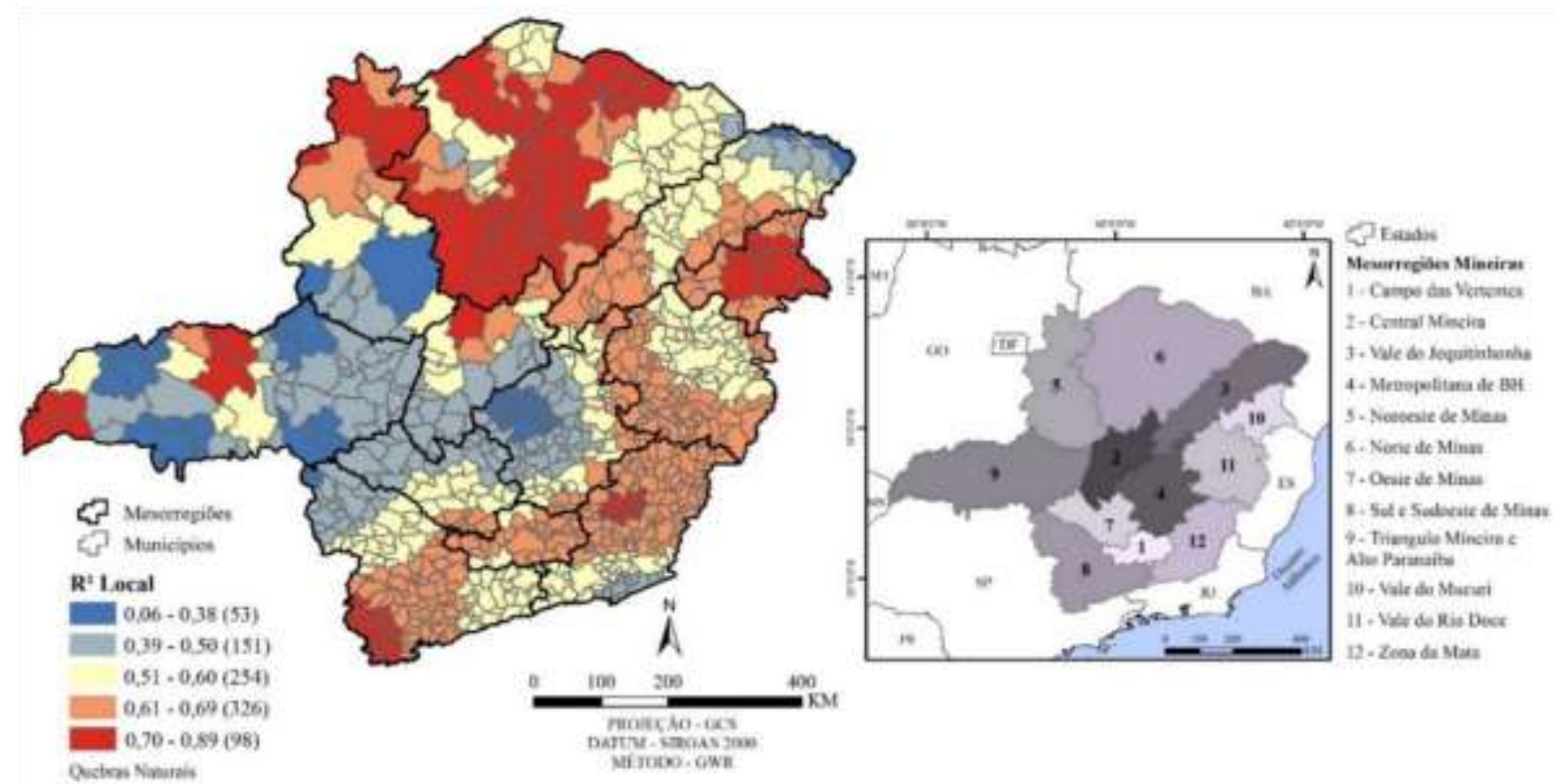

Fonte: Atlas do Desenvolvimento Humano no Brasil - 2013 e IBGE 2010.

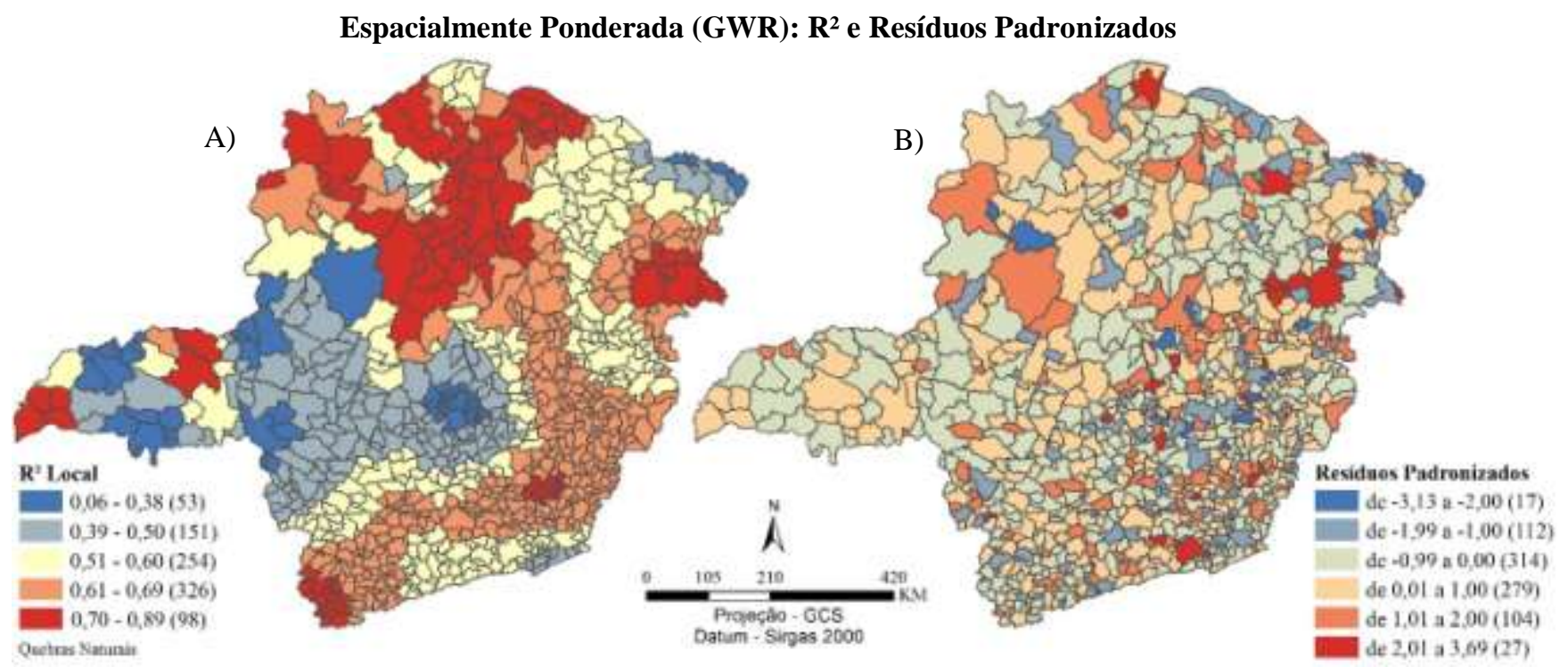

Fonte: Atlas do Desenvolvimento Humano no Brasil - 2013 e IBGE 2010.

Figura 3 - ICV e Renda média per capita nos municípios de Minas Gerais, 2010 - Regressão

Como o modelo teve um bom ajuste tanto na escala global quanto na local, os resíduos também foram baixos e não apresentam agrupamentos em sua distribuição ${ }^{2}$, como

\footnotetext{
${ }^{2}$ Foi realizado um teste utilizando o Índice de Moran para avaliar se existe algum padrão na distribuição dos resíduos. Os resultados demonstram que a distribuição é aleatória e sem a formação de agrupamentos ou padrões de dispersão (Índice de Moran Global: -0,01; Escore-Z: -1,12; P-Valor: 0,26).
} 
indicado na Figura 3B. Aqueles municípios que apresentam desvios inferiores a 2 (17), os seus valores de ICV são significativamente inferiores ao esperado, dado a sua renda média. Já nos casos contrários, em que os desvios são superiores a 2 (27), o ICV observado é superior ao esperado, ou seja, a renda é alta e como existe relação entre ICV e renda, estes municípios deveriam apresentar melhores valores para o ICV. Um exemplo pode ser o município de Juiz de Fora que apresenta renda média de $\mathrm{R} \$ 1.050,00$ e seu ICV é de 0,12.

\section{5-CONSIDERAÇÕES FINAIS}

Como mencionado anteriormente, a estatística espacial aplicada aos estudos socioeconomicos e ambientais pode ser bastante útil em diversas frentes. Com base no objetivo e na metodologia aplicada, percebe-se que existe uma forte correlação negativa ${ }^{3}$ entre alguns indicadores sociais e a renda nos municípios mineiros. Sendo assim, a hipótese inicial foi rejeitada e assume-se que, no geral, a partir dos resultados obtidos, a renda se materializa em melhores condições de vida sim.

Em relação às mesorregiões, nota-se que existe uma forte disparidade entre elas. $\mathrm{O}$ Triângulo Mineiro/Alto Paranaíba se destaca tendo em vista sua forte economia relacionada às atividades agropecuárias. O Norte de Minas e os Vales do Jequitinhonha e Mucuri apresentamse com as mesorregiões com maiores necessidades de investimento e desenvolvimento socioeconômico.

Por fim, sugere-se que estudos mais aprofundados sejam realizados tendo como recorte espacial os municípios que apresentaram maiores resíduos nesta análise. Seria interessante compreender melhor a dinâmica social e econômica destes locais, além dos motivos que geraram estes resultados. Além disso, uma análise futura semelhante à esta, utilizando os dados do próximo Censo Demográfico (2020), pode mostrar a evolução e as possíveis transformações dos municípios e mesorregiões mineiras no contexto socioeconômico dos últimos 10 anos do século XXI (2010-2010).

\footnotetext{
${ }^{3}$ De acordo com Rogerson (2012), nas correlação negativas entre duas variáveis, sempre que uma aumenta, a outra sempre diminui.
} 


\section{REFERÊNCIAS BIBLIOGRÁFICAS}

ARAÚJO, Edna Maria.; COSTA, Maria da Conceição.; OLIVEIRA, Nelson.; SANTANA, Francisco.; BARRETO, Maurício Lima.; HOGAN, Vivian.; ARAÚJO, Tânia. Distribuição espacial da mortalidade por homicídio e desigualdades sociais segundo a raça/cor em um espaço intra-urbano no Brasil. Revista Brasileira de Epidemiologia, v. 13, n. 4, pp. 549-560, 2010.

ARAÚJO, Everton.; URIBE-OPAZO, Miguel.; JOHANN, Jerry Adriani. Modelo de regressão espacial para estimativa da produtividade da soja associada a variáveis agrometeorológicas na região oeste do estado do Paraná. Engenharia Agrícola, v. 34, n. 2, p. 286-299, Jaboticabal, 2014.

ASSUNÇÃO, Renato. Estatística espacial aplicada. Disponível em: $<$ http://www.leg.ufpr.br/lib/exe/fetch.php/pessoais:renato:espacial:semana1.pdf>. Acesso em: 22 nov. 2017.

ATLAS DE DESENVOLVIMENTO HUMANO NO BRASIL 2013. Disponível em: <http://www.pnud.org.br/atlas/>. Acesso em 20 nov. 2017.

BARBETTA, Pedro Alberto. Estatística aplicada às ciências sociais. 8. Ed. Santa Catarina: Editora da UFSC, 2012, 318p.

CÂMARA, Gilberto; CASANOVA, Marco.; HEMERLY, Andrea.; MAGALHÃES, Geovane.; MEDEIROS, Claudia. Anatomia de sistemas de informações geográficas. Rio de Janeiro: Cartgraf LTDA, 1996, 205p.

GEARY, Roy. The contiguity ratio and statistical maping. The Incorporates Satistician, v. 5, pp. 115-145, 1954.

GONÇALVES, Annelise.; COSTA, Maria da Conceição.; BRAVA, José Uéreles. Análise da distribuição espacial da mortalidade neonatal e de fatores associados, em Salvador, Bahia, Brasil, no período 2000-2006. Cadernos de Saúde Pública, v. 27, n. 8, pp. 1581-1592, 2011.

WICKHAM, Hadley ggplot2: Elegant Graphics for Data Analysis. Springer-Verlag New York, 2009.

IGNÁCIO, Sergio Aparecido. Importância da estatística para o processo de conhecimento e tomada de decisão. Revista Paranaense de Desenvolvimento. Curitiba, n. 118, p. 175-192, 2010.

INSTITUTO BRASILEIRO DE GEOGRAFIA E ESTATÍSTICA - IBGE. Catalogo de download - geociências. Disponível em: < https://downloads.ibge.gov.br/downloads_geocien cias.htm>. Acesso em: 21 nov. 2017.

Informações sociais, demográficas e econômicas. Disponível em: <https://www.ibge.gov.br/institucional/o-ibge.html >. Acesso em: 19 nov. 2017. 
Resultados do Censo Demográfico de 2010. Disponível em:

$<$ https://www.ibge.gov.br/estatisticas-novoportal/sociais/populacao/9662-censodemografico2010.html?\&t=resultados >. Acesso em: 22 nov. 2017.

JÚNIOR, Noé Gonçalves Maranduba. Política regional, crescimento econômico e convergência de renda em Minas Gerais. Dissertação (Mestrado em Economia) Universidade Federal de Juiz de Fora, Juiz de Fora, 2007.

KREMPI, Ana Paula. Explorando recursos de estatística espacial para análise da acessibilidade da cidade de Bauru. Dissertação (Mestrado em Engenharia Civil) Universidade de São Paulo, São Carlos, 2004.

LOBO, Carlos.; FONSECA, Diego Ferreira.; GARCIA, Ricardo Alexandrino. Verticalização e permeabilização do solo urbano nos setores censitários de Belo Horizonte/MG. Rev. Bras. Estudos Urbanos e Regionais, v. 17, n. 3, p. 215-228, Recife, 2015.

MAGALHÃES, Monica de Avelar.; MEDRONHO, Roberto. Análise espacial da Tuberculose no Rio de Janeiro no período de 2005 a 2008 e fatores socioeconômicos associados utilizando microdado e modelos de regressão espaciais globais. Ciência \& Saúde Coletiva, v. 22, n. 3, pp. 381-849, 2017.

MORAN, Patrick Alfred Pierce. The interpretation of statistical maps. Journal of the Royal Statistic Society B, v. 10, p. 243-251.

RESENDE, Guilherme Mendes. O crescimento econômico dos municípios mineiros tem sido pró-pobre? Uma análise para o período 1991-2000. Nova Economia, v. 18, n. 1, pp. 119-154, 2008.

ROGERSON, Peter. Métodos estatísticos para geografia: um guia para o estudante. 3. Ed. Porto Alegre: Bookman, 2012, 348p.

ROSYCLER, Cristina Santos Simão. Distribuição de renda e pobreza no estado de Minas Gerais. Dissertação (Mestrado em Ciências - Economia Aplicada) - Universidade de São Paulo, Piracicaba, 2004.

SAWAYA, Ana Lydia.; SOLYMOS, Gisela.; MENEZES, Telma.; MARTINS, Paula. Os dois Brasis: quem são, onde estão e como vivem os pobres brasileiros. Estudos Avançados, v. 17, n. 48, pp. 21-45, 2003.

STUDENT. The elimination of spurious correlation due to position in time or space. Biometrika, v. 10, pp. 179-180, 1914. 